\title{
Majorana Fermions and Non-Abelian Statistics in Three Dimensions
}

\author{
Jeffrey C. Y. Teo and C. L. Kane \\ Department of Physics and Astronomy, University of Pennsylvania, Philadelphia, Pennsylvania 19104, USA
}

(Received 25 September 2009; published 25 January 2010)

\begin{abstract}
We show that three dimensional superconductors, described within a Bogoliubov-de Gennes framework, can have zero energy bound states associated with pointlike topological defects. The Majorana fermions associated with these modes have non-Abelian exchange statistics, despite the fact that the braid group is trivial in three dimensions. This can occur because the defects are associated with an orientation that can undergo topologically nontrivial rotations. A feature of three dimensional systems is that there are "braidless" operations in which it is possible to manipulate the ground state associated with a set of defects without moving or measuring them. To illustrate these effects, we analyze specific architectures involving topological insulators and superconductors.
\end{abstract}

DOI: 10.1103/PhysRevLett.104.046401

A fundamental feature of quantum theory is the quantum statistics obeyed by identical particles. For ordinary particles, Bose and Fermi statistics are the only possibilities. Emergent excitations in correlated many particle systems, however, can exhibit fractional [1-4] and non-Abelian [5] statistics. The simplest non-Abelian excitations, known as Ising anyons [6], are Majorana fermion states associated with zero energy modes that occur in the Bogoliubovde Gennes (BdG) description of a paired condensate [7]. They have been predicted in a variety of two dimensional (2D) electronic systems, including the $\nu=5 / 2$ quantum Hall effect [8], chiral $p$-wave superconductors (SCs) [9], and SC-topological insulator (TI) structures [10]. The ground state of $2 N$ Ising anyons has a $2^{N}$ degeneracy, and when identical particles are exchanged the state undergoes a non-Abelian unitary transformation [11,12]. Recent interest in non-Abelian statistics has been heightened by the proposal to use these features for topological quantum computation [13].

Fractional and non-Abelian statistics are usually associated with 2D because in 3D, performing an exchange twice is topologically trivial. In this Letter, we show that in 3D, Majorana fermion states are associated with pointlike topological defects, and that they obey non-Abelian exchange statistics, despite the triviality of braids. Our motivation came from the study of 3D SC-TI structures, where Majorana fermions arise in a variety of ways, such as (i) vortices at SC-TI interfaces [10], (ii) SC-magnet interfaces at the edge of a 2D TI [14-16], and (iii) band inversion domain walls along a SC vortex line. While the Majorana fermions in these cases can be identified using $1 \mathrm{D}$ or $2 \mathrm{D}$ effective theories, they must occur in a more general 3D theory. To unify them, we introduce a $\mathbb{Z}_{2}$ topological index that locates the zero modes in a generic 3D BdG theory. We then study a minimal 8-band model in which the defects can be understood as hedgehogs in a three component vector field. Ising non-Abelian exchange statistics arise because the hedgehogs have an orientation that can undergo nontrivial rotations. We will illustrate the
PACS numbers: 71.10.Pm, 03.67.Lx, 74.45.+c, 74.90.+n

intrinsic three dimensionality of the Majorana states by considering specific architectures involving SCs and TIs. A feature in 3D is the existence of "braidless" operations, in which the quantum information encoded in the Majorana states can be manipulated without moving or measuring [17] them.

To determine whether a Majorana mode is enclosed in a volume $V$, we topologically classify BdG Hamiltonians on $\partial V$, the 2D surface $V$. We assume the Hamiltonian varies slowly, so we can consider adiabatic changes as a function of two parameters $\mathbf{r}$ characterizing $\partial V$. The problem is then to classify particle-hole $(\mathrm{PH})$ symmetric $\mathrm{BdG}$ Hamiltonians $\mathcal{H}(\mathbf{k}, \mathbf{r})$, where $\mathbf{k}$ is defined in a $3 \mathrm{D}$ Brillioun zone (a torus $T^{3}$ ) and $\mathbf{r}$ is defined on a 2 -sphere $S^{2}$. PH symmetry is defined by an antiunitary operator $\Xi$ satisfying $\Xi^{2}=1$ and $\mathcal{H}(\mathbf{k}, \mathbf{r})=-\Xi \mathcal{H}(-\mathbf{k}, \mathbf{r}) \Xi^{-1}$. Assuming no other symmetries, this corresponds to class $\mathrm{D}$ of the general scheme $[18,19]$. Since $V$ may or may not enclose a zero mode, we expect a $\mathbb{Z}_{2}$ classification- a fact that can be established using methods of $K$ theory.

A formula for the topological invariant can be derived using a method similar to Qi, Hughes, and Zhang's [20] formulation of the invariant characterizing a 3D strong TI [21]. We introduce a one parameter deformation $\tilde{\mathcal{H}}(\lambda, \mathbf{k}, \mathbf{r})$ that adiabatically connects $\mathcal{H}(\mathbf{k}, \mathbf{r})$ at $\lambda=0$ to a trivial Hamiltonian independent of $\mathbf{k}$ and $\mathbf{r}$ at $\lambda=1$, while violating $\mathrm{PH}$ symmetry. $\mathrm{PH}$ symmetry can then be restored by including a mirror image $\tilde{\mathcal{H}}(\lambda, \mathbf{k}, \mathbf{r})=$ $-\Xi \tilde{\mathcal{H}}(-\lambda,-\mathbf{k}, \mathbf{r}) \Xi^{-1}$ for $-1<\lambda<0$. For $\lambda= \pm 1, \mathbf{k}$, $\mathbf{r}$ can be replaced by a single point, so the 6 parameter space $(\lambda, \mathbf{k}, \mathbf{r}) \sim \Sigma\left(T^{3} \times S^{2}\right)(\Sigma$ denotes the suspension $)$ has no boundary. $\tilde{\mathcal{H}}$ defined on this space is characterized by its integer valued third Chern character [22],

$$
\mathrm{Ch}_{3}[\mathcal{F}]=\frac{1}{3 !}\left(\frac{i}{2 \pi}\right)^{3} \int_{\Sigma\left(T^{3} \times S^{2}\right)} \operatorname{Tr}[\mathcal{F} \wedge \mathcal{F} \wedge \mathcal{F}] .
$$

Here, $\mathcal{F}=d \mathcal{A}+\mathcal{A} \wedge \mathcal{A}$ follows from the non-Abelian Berry's connection $\mathcal{A}_{i j}=\left\langle u_{i}|d| u_{j}\right\rangle$ associated with the 
negative energy eigenstates of $\tilde{\mathcal{H}}$. Because of PH symmetry, the contributions to (1) for $\lambda>0$ and $\lambda<0$ are equal. Moreover, since it is a total derivative, $\operatorname{Tr}\left[\mathcal{F}^{3}\right]=d \mathcal{Q}_{5}$ (omitting the $\wedge$ 's), where the Chern-Simons 5 form is [22]

$$
\mathcal{Q}_{5}=\operatorname{Tr}\left[\mathcal{A} \wedge(d \mathcal{A})^{2}+(3 / 2) \mathcal{A}^{3} \wedge d \mathcal{A}+(3 / 5) \mathcal{A}^{5}\right] \text {. }
$$

The integral over $\lambda>0$ can be then be pushed to the boundary $\lambda=0$ so that

$$
\mathrm{Ch}_{3}[\mathcal{F}]=\frac{2}{3 !}\left(\frac{i}{2 \pi}\right)^{3} \int_{T^{3} \times S^{2}} \mathcal{Q}_{5} .
$$

A different deformation $\tilde{\mathcal{H}}$ can change $\mathrm{Ch}_{3}[\mathcal{F}]$, but $\mathrm{PH}$ symmetry requires the change is an even integer. Likewise, the right hand side of (3) is only gauge invariant up to an even integer. The parity of (3) defines a $\mathbb{Z}_{2}$ topological invariant. We write it as

$$
\mu=\int_{S^{2}} \boldsymbol{\omega}(\mathbf{r}) \cdot d \mathbf{A} \quad \bmod 2
$$

where the gauge dependent Chern-Simons flux $\boldsymbol{\omega}=$ $\left(\omega^{1}, \omega^{2}, \omega^{3}\right)$ is defined by integrating out $\mathbf{k}$,

$$
\frac{1}{2} \epsilon_{i j k} \omega^{i}(\mathbf{r}) d x^{j} \wedge d x^{k}=\frac{1}{3}\left(\frac{i}{2 \pi}\right)^{3} \int_{T^{3}} Q_{5} .
$$

It is natural to associate $\mu$ with the presence of a zero mode - a fact that will be checked explicitly below.

We now introduce a minimal model that leads to an appealing physical interpretation for $\boldsymbol{\omega}(\mathbf{r})$. Since $\mu$ is based on $\mathrm{Ch}_{3}[\mathcal{F}]$, we expect a minimum of 8 bands is required. Consider a model parameterized by a three component vector field $\mathbf{n}$ of the form,

$$
\mathcal{H}=-i \gamma_{a} \partial_{a}+\Gamma_{a} n_{a}(\mathbf{r}) .
$$

Here, $\gamma_{a}$ and $\Gamma_{a}(a=1,2,3)$ are $8 \times 8$ Dirac matrices satisfying $\left\{\Gamma_{a}, \Gamma_{b}\right\}=\left\{\gamma_{a}, \gamma_{b}\right\}=2 \delta_{a b}$ and $\left\{\Gamma_{a}, \gamma_{b}\right\}=0$. $\mathcal{H}$ respects PH symmetry provided $\Xi \Gamma_{a} \Xi^{-1}=-\Gamma_{a}$ and $\Xi \gamma_{a} \Xi^{-1}=\gamma_{a}$. For $\mathbf{n}(\mathbf{r})=\mathbf{n}_{0}, \mathcal{H}$ has eigenvalues $E(\mathbf{k})= \pm\left(|\mathbf{k}|^{2}+\left|\mathbf{n}_{0}\right|^{2}\right)^{1 / 2}$ so that for $\mathbf{n}_{0} \neq 0$, there is a gap $2\left|\mathbf{n}_{0}\right|$. The seventh Dirac matrix $\gamma_{5} \equiv i \prod_{a} \gamma_{a} \Gamma_{a}$ is not an allowed mass term because $\Xi \gamma_{5} \Xi^{-1}=\gamma_{5}$. A more general Hamiltonian could also involve products of the Dirac matrices, but such Hamiltonians can be homotopically deformed to the form of (6) without closing the gap [19]. To regularize (6) at $|\mathbf{k}| \rightarrow \infty$, we include an additional term $\epsilon|\mathbf{k}|^{2} \Gamma_{3}$, so $\mathbf{k}$ can be defined on a compact Brillouin zone $S^{3}$. The analysis is simplest for $\epsilon \rightarrow 0$, where the low energy properties are isotropic in $\mathbf{n}$.

$\mathcal{H}$ can be physically motivated by considering a BdG Hamiltonian describing ordinary and topological insulators coexisting with superconductivity. The Dirac matrices are specified by three sets of Pauli matrices: $\vec{\tau}$ for PH space, $\vec{\sigma}$ for spin, and $\vec{\mu}$ for an orbital degree of freedom. We identify $\vec{\gamma}=\tau_{z} \mu_{z} \vec{\sigma}, \Gamma_{1}=\tau_{x}, \Gamma_{2}=\tau_{y}$, and $\Gamma_{3}=\tau_{z} \mu_{x}$, along with $\Xi=\sigma_{y} \tau_{y} K$. $\mathbf{n}$ is then $\left(\Delta_{1}, \Delta_{2}, m\right)$, where $\Delta=$ $\Delta_{1}+i \Delta_{2}$ is a SC order parameter and $m$ is a mass describing a band inversion. For $\Delta=0,(6)$ is a doubled version of the model for a 3D TI discussed in Ref. [20]. For $\epsilon>0$, $m>0$ describes a trivial insulator, while $m<0$ describes a TI with a band inversion near $\mathbf{k}=0$. An interface where $m$ changes sign corresponds to the surface of a TI, which has gapless surface states. Introducing $\Delta \neq 0$ to the interface then describes the proximity induced SC state [10].

To locate the zero modes, we take $\mathbf{n}(\mathbf{r})$ to vary slowly with $\mathbf{r}$ and evaluate $\boldsymbol{\omega}(\mathbf{r})$ using (1)-(5). This can be done by noting that $\mathcal{H}$ defines a 6 component unit vector given by the direction $\hat{\mathbf{d}}^{5}(\mathbf{k}, \mathbf{r})$ of $\left(k_{1}, k_{2}, k_{3}, n_{1}+\epsilon|\mathbf{k}|^{2}, n_{2}, n_{3}\right)$ on $S^{5}$. The deformed Hamiltonian $\tilde{\mathcal{H}}$ can be defined by adding $\lambda \gamma_{5}$ to $\mathcal{H}$ so that $\tilde{\mathcal{H}}$ defines a vector on $S^{6}$ given by $\hat{\mathbf{d}}^{6}=\left(\sqrt{1-\lambda^{2}} \hat{\mathbf{d}}^{5}, \lambda\right) . \mathrm{Ch}_{3}[\mathcal{F}]$ is the volume on $S^{6}$ swept out by $\hat{\mathbf{d}}^{6}(\lambda, \mathbf{k}, \mathbf{r})$. Then, $\int \mathcal{Q}_{5}$ is the volume in the "northern hemisphere" of $S^{6}$ swept out by $\hat{\mathbf{d}}^{5}(\mathbf{k}, \mathbf{r})$, which is confined to the "equator," $\lambda=0$. This is then related to the area on $S^{5}$ swept out by $\hat{\mathbf{d}}^{5}(\mathbf{k}, \mathbf{r})$. Performing the integral on $\mathbf{k}$ for $\epsilon \rightarrow 0$ gives

$$
\omega^{i}(\mathbf{r})=\frac{1}{8 \pi} \epsilon^{i j k} \hat{\mathbf{n}} \cdot \partial_{j} \hat{\mathbf{n}} \times \partial_{k} \hat{\mathbf{n}},
$$

where $\hat{\mathbf{n}}=\mathbf{n} /|\mathbf{n}|$. Thus, the topological charge that signals a zero mode inside $V$ is the parity of the $S^{2}$ winding number of $\hat{\mathbf{n}}$ on $\partial V$. Zero modes are associated with hedgehogs in $\hat{\mathbf{n}}(\mathbf{r})$. A simple example of a hedgehog is an SC vortex at the interface between a TI and an insulator. Though the hedgehog topological charge can be any integer, an even integer in (4) can be unwound by a $\mathbf{k}$ and $\mathbf{r}$ dependent gauge transformation.

The presence of a zero mode associated with a hedgehog can be demonstrated with a simple linear model $n_{a}(\mathbf{r})=$ $M_{a b} r_{b}$, which has a hedgehog with charge $\operatorname{sgn}(\operatorname{det}[M])$ at $\mathbf{r}=0$. This is solved by expressing $M$ in terms of its principle axes: $M=\mathcal{O}_{1}^{T} \operatorname{diag}\left(M_{1}, M_{2}, M_{3}\right) \mathcal{O}_{2}$, where $\mathcal{O}_{1}$ and $\mathcal{O}_{2}$ are orthogonal matrices that diagonalize $M M^{T}$ and $M^{T} M$, respectively. Defining $r_{a}^{\prime}=\mathcal{O}_{1 a b} r_{b}, n_{a}^{\prime}=$ $\mathcal{O}_{2 a b} n_{b}, \gamma_{a}^{\prime}=\mathcal{O}_{1 a b}^{T} \gamma_{b}$, and $\Gamma_{a}^{\prime}=\mathcal{O}_{2 a b}^{T} \Gamma_{b}$, it is straightforward to express $\mathcal{H}^{2}$ as three independent harmonic oscillators,

$$
\mathcal{H}^{2}=\sum_{a} M_{a}\left(2 n_{a}+1-\xi_{a}\right)
$$

where $n_{a}$ are oscillator quantum numbers and $\xi_{a}=i \gamma_{a}^{\prime} \Gamma_{a}^{\prime}$ are commuting operators. There is a single zero energy state with $n_{a}=0$ and $\xi_{a}=1$. This zero mode is the nondegenerate eigenstate with eigenvalue 3 of $\sum_{a} \xi_{a}=$ $i \sum_{a, b} \gamma_{a} \mathcal{O}_{a b} \Gamma_{b}$, where $\mathcal{O}=\mathcal{O}_{1} \mathcal{O}_{2}^{T}$.

A key feature of the zero mode is its dependence on the relative orientation $\mathcal{O}$ of the principle axes in $\mathbf{r}$ and $\mathbf{n}$ space. This can lead to a non trivial holonomy when $M$ (and hence $\mathcal{O}$ ) varies. We construct the zero mode by starting with $\left|\Psi_{0}\right\rangle$ which satisfies $\sum_{a} i \gamma_{a} \Gamma_{a}\left|\Psi_{0}\right\rangle=3\left|\Psi_{0}\right\rangle$ 
and then doing a unitary transformation that takes $\Gamma_{a}$ to $\mathcal{O}_{a b} \Gamma_{b}$. If we parameterize the rotation $\mathcal{O}$ with a vector $\boldsymbol{\Omega}$ specifying the axis and angle $|\boldsymbol{\Omega}| \leq \pi$, then

$$
|\Psi(\boldsymbol{\Omega})\rangle=e^{\epsilon_{a b c} \Gamma_{a} \Gamma_{b} \Omega_{c} / 4}\left|\Psi_{0}\right\rangle
$$

This gauge satisfies $\langle\Psi(\boldsymbol{\Omega}) \mid d \Psi(\boldsymbol{\Omega})\rangle=0$ for $|\boldsymbol{\Omega}|<\pi$ but is not globally defined because $|\Psi(\boldsymbol{\Omega})\rangle=-|\Psi(-\boldsymbol{\Omega})\rangle$ when $|\boldsymbol{\Omega}|=\pi$. This reflects the nontrivial topology of $S O(3)$, characterized by the homotopy $\pi_{1}[S O(3)]=\mathbb{Z}_{2}$. When $\mathcal{O}$ varies along a nontrivial loop in $S O(3)$, the wave function of the zero mode changes sign. The associated Majorana operator $\gamma_{i}$ (not to be confused with the Dirac matrix $\gamma_{a}$ ) also changes sign. This is one of our central results, and it is this fact that allows Ising non-Abelian statistics in 3D. A simple example of a nontrivial loop is the $2 \pi$ rotation that occurs when the SC phase advances by $2 \pi$.

Though we derived it with the linear 8 band model, our conclusion that there is nontrivial holonomy for defects is more general. A formulation based on (4) will appear elsewhere. In the 8-band model, a general defect history is characterized by $\mathbf{n}(\mathbf{r}, t)$, where $\mathbf{r}$ is on a surrounding surface $S^{2}$ and $t$ varies on a closed path $S^{1}$. These are classified by the homotopy of maps $S^{2} \times S^{1} \rightarrow S^{2}$, which were first analyzed by Pontrjagin [23], and have appeared in other physical contexts [24]. When the hedgehog's topological charge is $\pm p$, the classification is $\mathbb{Z}_{2 p}$. This is related to the integer Hopf invariant for maps $S^{3} \rightarrow S^{2}$ which applies when $p=0$. Like the Hopf invariant, it can be understood in terms of the linking of curves in $S^{2} \times S^{1}$ [25]. The discussion below resembles the analysis of Wilczek and Zee [2] of the statistics of Skyrmions in the $(2+1) D$ nonlinear $\sigma$ model with a Hopf term.

To study the exchange statistics of the Majorana modes, we consider the adiabatic evolution of the state when they are exchanged. We thus consider a $3+1 D$ history $\hat{\mathbf{n}}(\mathbf{r}, t)$ satisfying $\hat{\mathbf{n}}(\mathbf{r}, T)=\hat{\mathbf{n}}(\mathbf{r}, 0)$ with hedgehogs at $\left\{\mathbf{r}_{i}(t)\right\}$ with $\mathbf{r}_{1(2)}(T)=\mathbf{r}_{2(1)}(0)$. To visualize $\hat{\mathbf{n}}(\mathbf{r}, t)$, it is useful to consider the inverse image paths in $\mathbf{r}$ space that map to two specific points on $S^{2}$. Such paths begin and end on hedgehogs, and a crucial role will be played by their linking properties. Figure 1 depicts four hedgehogs, where the top two (positive) hedgehogs are interchanged. At the first step in (b), the locations of the hedgehogs have been interchanged. Since the inverse image paths have been "dragged," $\hat{\mathbf{n}}(\mathbf{r})$ is not the same as its original configuration. Panels (c)-(f) show a sequence of smooth deformations that untangle $\hat{\mathbf{n}}(\mathbf{r})$. The key point is that the two paths (which map to different points on $S^{2}$ ) can never cross each other. However, a path can cross itself and "reconnect," as in (c), (d), (e). The deformations from (a)-(e) preserve the orientation of the hedgehogs, but leave behind a twist. To return $\hat{\mathbf{n}}(\mathbf{r})$ to its original configuration in (f) requires a $2 \pi$ rotation of one of the hedgehogs. This results in an interchange rule for the Majoranas,
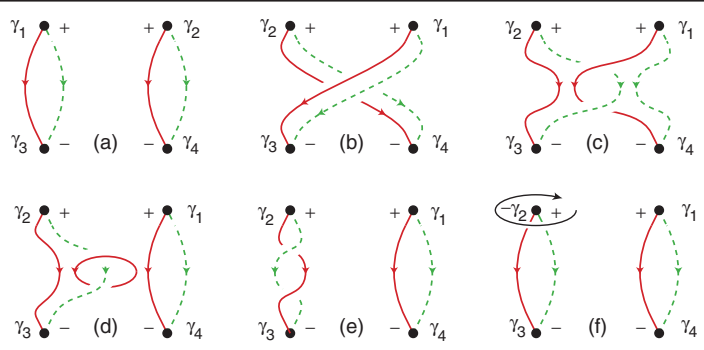

FIG. 1 (color online). Inverse image paths depicting the interchange of two hedgehogs, as described in the text. (a)-(e) show a sequence of continuous deformations of $\mathbf{n}(\mathbf{r})$ that preserve the orientation of the hedgehogs. In (f), a $2 \pi$ rotation is required to return to the original configuration.

$$
T_{12}: \gamma_{1} \rightarrow \gamma_{2} ; \quad \gamma_{2} \rightarrow-\gamma_{1}
$$

analogous to the rules $[11,12]$ for braiding vortices in $2 \mathrm{D}$ and can be represented by $T_{12}=\exp \left[\pi \gamma_{1} \gamma_{2} / 4\right]$. Note that in (d), (e), the twist could have been left on the other side, which would have led to $T_{21}=T_{12}^{\dagger}$. The two choices for $T_{12}$ correspond to physically distinct interchange trajectories that generalize the right- and left-handed braiding operations in 2D.

Performing the same interchange twice leads to a nontrivial operation, since $T_{i j}^{2}=\gamma_{i} \gamma_{j}$ changes the sign of both $\gamma_{i}$ and $\gamma_{j}$. This is natural in 2D because it is a noncontractable braid. In $3 \mathrm{D}$, however, $T_{i j}^{2}$ can be smoothly deformed into an operation in which all particles are held fixed. Thus, there is an operation, specified by a history $\hat{\mathbf{n}}(\mathbf{r}, t)$, that rotates any pair of stationary hedgehogs by $2 \pi$, and implements the operation $\gamma_{i} \gamma_{j}$. The existence of such "braidless" operations is a feature of Ising non-Abelian statistics in 3D. Although these operations form an admittedly limited Abelian subgroup, they nonetheless offer a method for manipulating the quantum information encoded in the Majorana fermions without moving or measuring them.

We now illustrate these effects using specific architectures involving TIs and SCs. It is easiest to engineer Majorana modes using structures involving interfaces or vortex lines, where $\boldsymbol{\omega}$ is confined to lines or planes. Nonetheless, such structures can exhibit intrinsically 3D effects. Consider first the structure in Fig. 2(a), which involves two disconnected spherical TIs surrounded by a SC and connected to each other by a Josephson junction. Suppose that each sphere has a single \pm pair of vortices so that there are 4 Majorana states on the spheres. The internal state of the Majorana fermions can be represented in a basis of eigenstates of $n=i \gamma_{1} \gamma_{2}$ and $n^{\prime}=i \gamma_{3} \gamma_{4}$. For an isolated system, the parity of $n+n^{\prime}$ is fixed so the system is a single qubit with basis vectors $\left|n n^{\prime}=00,11\right\rangle$. The state can be initialized and measured in this basis with a probe that couples to both $\gamma_{1}$ and $\gamma_{2}$. Suppose $\gamma_{3}$ is adiabatically transported around $\gamma_{1}$ as shown. This is similar to a $2 \mathrm{D}$ braid, and it implements $T_{13}^{2}$, which interchanges $|00\rangle$ and 


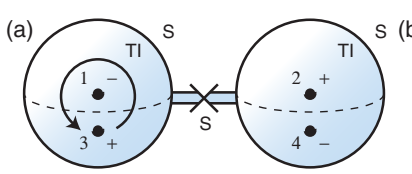

(c)

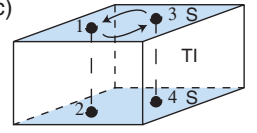

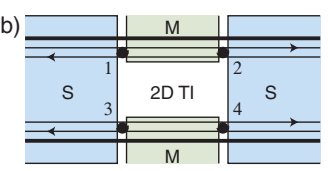

(d)

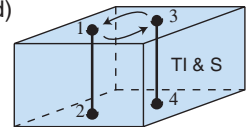

FIG. 2 (color online). Architectures demonstrating 3D Majorana states using SCs and TIs. (a) Braiding 3 around 1 can be deformed into a braidless operation. (b) A geometry for implementing braidless operations with a Josephson junction device [15]. (c), (d) Thin film geometries for interchanging and measuring Majorana states. (c) shows a layered SC-TI-SC structure, while (d) shows a thin film of a SC weakly doped TI.

|11〉. Note, however, that this "braid" can be smoothly contracted to zero by sliding the path around the other side of the sphere. In the process, however, the path crosses the junction connecting the spheres, resulting in a $2 \pi$ phase slip. Thus, the braiding operation can be smoothly deformed into a "braidless" operation, where $\gamma_{1}, \gamma_{3}$ are held fixed, but the phase difference between the two SCs advances by $2 \pi$.

A similar but more feasible version of the braidless operation occurs for the Josephson junction structure in Fig. 2(b), which involves Majorana modes at a SC-magnet interface at the edge of a 2D TI. As argued in Ref. [15], when the phase difference across the junction is advanced by $2 \pi$, the fermion parity associated with $i \gamma_{1} \gamma_{2}$ changes, resulting in a fractional Josephson effect.

Figure 2(c) shows a TI coated on the top and bottom with SC films. In a magnetic field, Majorana states occur at vortices on both the top and bottom. If the SC films are thinner than the penetration depth, the field is constant, so the top and bottom vortices are independent. If the TI is thin, there will be a weak vertical coupling that splits nearby Majorana modes according to $n=i \gamma_{1} \gamma_{2}$ and $n^{\prime}=$ $i \gamma_{3} \gamma_{4}$. The states $\left|n n^{\prime}\right\rangle$ will have slightly different charges, which may allow $n, n^{\prime}$ to be measured with a sensitive charge detector. Suppose the state is initially $|00\rangle$. Interchanging $\gamma_{1}, \gamma_{3}$ on the top keeping the bottom fixed leads to the entangled state $(|00\rangle+|11\rangle) / \sqrt{2}$. A variant on this geometry [Fig. 2(d)] is a thin film of a bulk SC weakly doped TI, in which the surface states acquire SC similar to the proximity induced state. In this case, the interchange of $\gamma_{1}, \gamma_{3}$ involves a reconnection of the vortex lines (which in principle have a finite energy gap) connecting them.

Recently, SC has been observed in $\mathrm{Cu}_{x} \mathrm{Bi}_{2} \mathrm{Se}_{3}$ for $x \sim 0.15$ [26]. It will be interesting to determine whether this material is in the weakly doped regime, with Majorana modes at the ends of vortex lines, or a more conventional
SC, which could be used in Fig. 2(c). There are certainly technical challenges associated with manipulating the vortices and measuring their charge state. Nonetheless, we hope that the prospect of detecting 3D non-Abelian statistics in such a system will provide motivation for further exploration.

We thank Liang Fu for insightful discussions and Bryan Chen and Randy Kamien for introducing us to the Pontrjagin invariant. This work was supported by NSF Grant No. 0906175.

[1] J. M. Leinaas and J. Myrheim, Nuovo Cimento Soc. Ital. Fis. B 37, 1 (1977).

[2] F. Wilczek and A. Zee, Phys. Rev. Lett. 51, 2250 (1983).

[3] B. I. Halperin, Phys. Rev. Lett. 52, 1583 (1984).

[4] D. Arovas, J. R. Schrieffer, and F. Wilczek, Phys. Rev. Lett. 53, 722 (1984).

[5] G. Moore and N. Read, Nucl. Phys. B360, 362 (1991).

[6] C. Nayak et al., Rev. Mod. Phys. 80, 1083 (2008).

[7] N. Read and D. Green, Phys. Rev. B 61, 10267 (2000).

[8] M. Greiter, X. G. Wen, and F. Wilczek, Nucl. Phys. B374, 567 (1992)

[9] S. Das Sarma, C. Nayak, and S. Tewari, Phys. Rev. B 73, 220502(R) (2006).

[10] L. Fu and C. L. Kane, Phys. Rev. Lett. 100, 096407 (2008).

[11] C. Nayak and F. Wilczek, Nucl. Phys. B479, 529 (1996).

[12] D. A. Ivanov, Phys. Rev. Lett. 86, 268 (2001).

[13] A. Kitaev, Ann. Phys. (N.Y.) 303, 2 (2003).

[14] A. Kitaev, arXiv:cond-mat/0010440.

[15] L. Fu and C. L. Kane, Phys. Rev. B 79, 161408(R) (2009).

[16] J. Nilsson, A. R. Akhmerov, and C. W. J. Beenakker, Phys. Rev. Lett. 101, 120403 (2008).

[17] P. Bonderson, M. Freedman, and C. Nayak, Phys. Rev. Lett. 101, 010501 (2008).

[18] A. P. Schnyder, S. Ryu, A. Furusaki, and A. W. W. Ludwig, Phys. Rev. B 78, 195125 (2008); AIP Conf. Proc. 1134, 10 (2009).

[19] A. Kitaev, AIP Conf. Proc. 1134, 22 (2009); arXiv:0901.2686.

[20] X. L. Qi, T. L. Hughes, and S. C. Zhang, Phys. Rev. B 78, 195424 (2008).

[21] L. Fu, C. L. Kane, and E. J. Mele, Phys. Rev. Lett. 98, 106803 (2007); J. E. Moore and L. Balents, Phys. Rev. B 75, 121306(R) (2007).

[22] M. Nakahara, Geometry, Topology and Physics (Adam Hilger, Bristol, 1990).

[23] L. S. Pontrjagin, Rec. Math. [Mat. Sbornik] N.S. 9, 331 (1941); http://mi.mathnet.ru/eng/msb6073.

[24] J. Jäykkä and J. Hietarinta, Phys. Rev. D 79, 125027 (2009).

[25] L. Kapitanski, in London Mathematical Society Durham Symposium (unpublished); http://www. maths.dur.ac.uk/ events/Meetings/LMS/200/OTSA/.

[26] Y.S. Hor et al., Phys. Rev. Lett. (to be published). 